\title{
Pathologische Frakturen bei Osteoporose
}

\author{
Tobias Vogel, Wolf Mutschler
}

\section{Zusammenfassung}

In der Bundesrepublik Deutschland leben rund 6000000 Menschen mit einer Osteoporose. Etwa 50\% dieser Patienten haben bereits eine oder mehrere Frakturen erlitten. Die Osteoporose wird als systemische Erkrankung des Skeletts mit Reduzierung der Knochenfestigkeit und daraus folgendem hohen Frakturrisiko definiert. Pathologische Frakturen sind definiert als Frakturen, die an einem aus systemischen oder lokalen Gründen krankhaft vorgeschädigten und mechanisch vermindert belastbaren Knochen eintreten. Osteoporotische Knochenbrüche stellen also die häufigste Art pathologischer Frakturen dar. Durch die demografische Entwicklung in den industrialisierten Ländern wird sich die Anzahl dieser Frakturen in den nächsten Jahren weiter erhöhen. Neben der konservativen Behandlung stabiler, nicht dislozierter Frakturen besitzt die operative Stabilisierung einen hohen Stellenwert in der Therapie dieser häufigen Frakturen. Ziel ist die rasche Wiederherstellung der Mobilität des betroffenen Skelettabschnitts, da hiermit die frakturassoziierte Morbidität und Mortalität gesenkt werden kann. Voraussetzung hierfür ist eine belastungsfähige Pri- märstabilität der Osteosynthese. Sie soll es dem Patienten ermöglichen, bereits in den ersten Tagen nach der operativen Versorgung, mit der krankengymnastischen Beübung zu beginnen. Neben den bewährten Verfahren wie z.B. Marknagelosteosynthese, Plattenosteosynthese und dem Einsatz der Endoprothetik existiert eine Reihe neuer und innovativer Verfahren zur Frakturstabilisierung. Winkelstabile Implantate, minimalinvasive Osteosynthesen und die Vertebro- und Kyphoplastie osteoporotischer Wirbelkörperfrakturen seien hierfür als Beispiele genannt. Die Entwicklung biodegradierbarer Knochenfüllstoffe und moderner Osteosyntheseverfahren durch die experimentelle Unfallchirurgie und Biomechanik soll in Zukunft der Verbesserung der Versorgungssituation von Patienten mit osteoporosebedingten Frakturen dienen.

\section{Pathological Fractures and Osteoporosis}

In Germany about 6000000 patients with osteoporosis were reported in $2004,50 \%$ of whom have already suffered at least one fracture. Osteoporosis is currently defined as a systemic skeletal disease characterised by low bone mass and microarchitectural deterioration of bone tissue, with a consequent increase in bone fragility and susceptibility to fracture. Osteoporotic fractures therefore represent the most frequent kind of pathological fracture. As a result of our aging society the number of fragility fractures will rise enormously within the next 20 years. Besides conservative treatment, surgery is the most common means of fracture stabilisation. The principal object of fracture surgery is the immediate restoration of mobility of the fractured extremity to reduce fracture-associated morbidity and mortality, especially in the older patient. This requires a primary weight-bearing stability of the osteosynthesis to allow early physical therapy. In addition to conventional procedures such as intramedullary nailing, plates or joint replacements, new and innovative stabilisation procedures, e.g., angle-stable plates, minimally invasive surgery or vertebroplasty and kyphoplasty have become more and more acknowledged. The development of biodegradable bone cements and the advancement of osteosynthesis procedures are necessary for modern fracture treatment in patients with fragility fractures.

\section{Einleitung}

Pathologische Frakturen sind definiert als Frakturen, die an einem aus systemischen oder lokalen Gründen krankhaft vorgeschädigten und mechanisch vermindert belastbaren Knochen eintreten $[1,12]$.

Typische Beispiele für Erkrankungen, die pathologische Frakturen begünsti-

OP-JOURNAL 2006; 22: 16-20

(c) Georg Thieme Verlag KG Stuttgart · New York gen, sind primäre Knochentumoren (z.B. Osteosarkom, Osteom), Metastasen (z.B. Mama-CA, Prostata-CA, Nierenzell-CA) und endokrine Erkrankungen(z.B.Hyperparathyreoidismus, Osteomalazie).

Die WHO definiert die Osteoporose als „... eine systemische Erkrankung des Skeletts, die durch eine erniedrigte Knochenmasse und eine Verschlechterung der Mikroarchitektur des Knochens gekennzeichnet ist. Daraus folgt eine zunehmende Brüchigkeit und ein gehäuftes Auftreten von Frakturen“ [17].
Somit erfüllen osteoporosebedingte Frakturen die Kriterien pathologischer Frakturen, sie stellen sogar die häufigste pathologische Fraktur überhaupt dar. Dem Unfallchirurgen kommt in der Behandlung osteoporotischer Frakturen und der Einleitung von Diagnostik und Therapie der Osteoporose die Schlüsselrolle zu $[2 ; 15]$.

Die Einteilung der Osteoporose erfolgt nach unterschiedlichen Gesichtspunkten. Klinisch bewährt hat sich die Einteilung in eine primäre und eine sekundäre Os- 
teoporose, da sich hieraus direkte therapeutische Konsequenzen ableiten lassen.

Unter der primären Osteoporose versteht man die idiopathische, postmenopausale und altersassoziierte Osteoporose. Sie stellt die weitaus häufigste Erscheinungsform der Osteoporose dar und hält einen Anteil von rund 95\% aller Osteoporoseformen. Sekundäre Osteoporosen sind die Folge anderer Erkrankungen des Stoffwechsels oder des Skelettsystems oder aber die Folge langjähriger Einnahme bestimmter Medikamente. Typische Beispiele hierfür sind die Inaktivitätsosteoporose, die Osteoporose bei Hyperparathyreoidismus oder die kortisoninduzierte Osteoporose. Allen Formen gemeinsam sind die messbare Reduktion der Knochendichte sowie die damit einhergehende hohe Frakturanfälligkeit. Knochen mit einem hohen Anteil an Spongiosa sind besonders häufig und zeitlich früher betroffen. Hierzu zählen neben den Wirbelkörpern v.a. die epi- und metaphysären Bereiche von proximalem Femur und Humerus sowie der distale Radius.

\section{Hauptteil}

\section{Diagnostik}

Das diagnostische Vorgehen zur Identifikation osteoporosebedingter, pathologischer Frakturen unterscheidet sich nicht vom Vorgehen bei anderen Frakturen. Am Anfang steht neben der genauen Unfallanamnese, bei der besonderer Wert auf den Unfallmechanismus und die einwirkenden Kräfte, frühere Frakturen und die Begleitmedikation gelegt wird, die körperliche Untersuchung. Vor allem Hyperkyphose, Klopfschmerzhaftigkeit der Wirbelsäule, eine Abnahme der Körpergröße $>4 \mathrm{~cm}$ und frühere Frakturen können hierbei richtungweisend für eine der Fraktur zugrunde liegende Osteoporose sein.

\section{Bildgebende Diagnostik}

\section{Frakturnachweis}

Den wichtigsten Pfeiler der Frakturdiagnostik stellt nach wie vor das konventionelle Röntgenbild der betroffenen Körperregion in zwei Ebenen dar. Hiermit kann der größte Teil der Extremitätenfrakturen sicher identifiziert und klassifiziert werden. Die Computertomographie (CT) findet ihren Einsatz bei den bei Osteoporose besonders häufig auftretenden Wirbelkörperfrakturen. Sie dient

Tab. 1 WHO Definition der Osteoporose anhand der Ergebnisse der Knochendichtemessung (DXA). SD = Standardabweichung

\begin{tabular}{ll} 
Bewertung & T-Score \\
\hline Normalbefund & $\geq-1 \mathrm{SD}$ \\
Osteopenie & -1 bis $-2,5 \mathrm{SD}$ \\
Osteoporose (messtechnisch) & $\leq-2,5 \mathrm{SD}$ \\
Osteoporose (manifest) & $\leq-2,5 \mathrm{SD}$ plus Fraktur
\end{tabular}

neben der Frakturklassifikation vor allem der Stabilitätsbeurteilung. Da viele Patienten bereits mehr als eine Wirbelkörperfraktur erlitten haben, die klinischen Beschwerden häufig unspezifisch sind und das konventionelle Röntgenbild selten in der Lage ist, die Frakturen hinsichtlich ihres zeitlichen Auftretens zu unterscheiden, ist der Einsatz der Skelettszintigraphie mithilfe $99 \mathrm{mTc}$-markierter Bisphosphonate oder der Magnetresonanztomographie (MRT) hilfreich. Der Vorteil der Szintigraphie liegt dabei in einer raschen Beurteilung des gesamten Skeletts. Herdförmige Mehranreicherungen im Bereich der Wirbelsäule deuten auf Frakturen oder auf degenerative, entzündliche oder neoplastische Läsionen hin. Bereits zwei Tage nach einer Fraktur ist eine Anreicherung zu erwarten. Die Nachteile der Szintigraphie liegen neben der Strahlenbelastung in der begrenzten Fähigkeit der strukturellen Darstellung. Dies bleibt weiteren bildgebenden Verfahren vorbehalten.

Mittels der MRT lässt sich frei von jeglicher Strahlenbelastung eine Differenzierung von metastatischen und osteoporotischen Frakturen vornehmen.

Außerdem gelingt mit ihrer Hilfe die zweifelsfreie Einteilung in alte und frische Frakturen. Durch spezielle Gradientenechosequenzen und die Applikation von Kontrastmittel lässt sich die Darstellungsqualität und damit die diagnostische Aussagekraft noch erheblich verbessern.

\section{Osteoporosenachweis}

An erster Stelle und von der WHO zur Osteoporosediagnostik empfohlen steht die Kombination aus konventioneller Radiographie und Knochendichtemessung mittels Dual-X-ray-Absorptiometrie (DXA).

Die DXA-Messung ist heute die ausgereifteste und populärste Messmethode.

Sie wird von der WHO favorisiert und stellt die Grundvoraussetzung einer stan- dardisierten Osteoporosediagnostik dar. Neben den Vorteilen einer sehr geringen Strahlenbelastung (1-3 mREM) und einer unkomplizierten, schnellen und preiswerten Durchführbarkeit stellt sie keine Belastung für den Patienten dar. Sie misst sehr genau (Richtigkeit 2-6\%, Präzision $1-3 \%$ ) und ist dadurch ideal für Kontrollmessungen geeignet [2;3]. Die Messung erfolgt definitionsgemäß an Hüfte und LWS. Ermittelt wird der sog. „T-Score“. Es handelt sich dabei um den Vergleich der Dichtewerte der gemessenen Person mit denen eines gesunden jungen Erwachsenen (Vergleich mit der maximalen Knochendichte), angegeben als Standardabweichung. Anhand der ermittelten TWerte erfolgt definitionsgemäß die Diagnosestellung der Osteoporose (Tab.1) [17].

\section{Therapie der Frakturen}

\section{Konservativ}

Die konservative Therapie osteoporotischer Frakturen hat bei nicht dislozierten und stabilen Extremitätenfrakturen sowie bei stabilen Kompressionsfrakturen der Wirbelkörper eine Berechtigung.

Auch die Begleiterkrankungen, der funktionelle Anspruch des individuellen Patienten und das Schmerzniveau müssen bei der Entscheidung zur konservativen Frakturbehandlung mit in Betracht gezogen werden. Außerdem muss sorgfältig zwischen der zu erwartenden Morbidität und Mortalität der alternativ zur Verfügung stehenden operativen Therapie und der konservativen Therapie mittels Immobilisation, Physiotherapie und medikamentöser Analgesie abgewogen werden. Vor allem die bei Osteoporose häufigen Kompressionsfrakturen der Wirbelkörper sollten in Abhängigkeit der Stabilität zunächst konservativ behandelt werden. Erst wenn die o.g. Maßnahmen über 4-6 Wochen keinen Erfolg zeigen und die Patienten trotz ausreichender Analgesie weiterhin unter starken Schmerzen leiden, kommen operative oder interventionelle Verfahren zur Stabilisation der Fraktur zum Einsatz. 

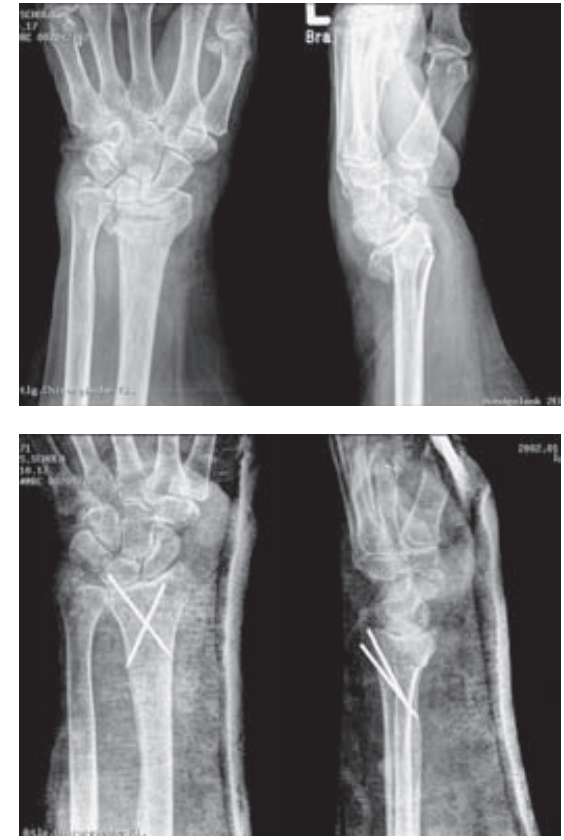

Abb. 1 Distale Radiusfraktur und Versorgung mittels minimalinvasiver Spickdrahtosteosynthese.

\section{Operativ}

Ziel der operativen Therapie osteoporotischer Frakturen ist das Erreichen einer übungs- und möglichst belastungsstabilen Primärstabilität. Hierunter versteht man die durch das Osteosyntheseverfahren und die Wahl des Osteosynthesematerials gegebene Stabilität zwischen der Knochensubstanz und dem Implantat. Die Primärstabilität hat entscheidenden Einfluss auf die Nachbehandlung und soll dem Patienten eine frühe krankengymnastische Beübung der betroffenen Extremität ermöglichen. Vor allem die in der Frühphase der Erkrankung auftretende und deutliche Reduktion an spongiösem Knochen, aber auch die später folgende Minderung der Festigkeit der Kortikalis, stellt uns oft vor große Probleme bei der Implantatverankerung. Ein weiteres Problem stellt die sekundäre Überlastung der Implantate durch eine verzögerte Knochenbruchheilung bei gleichzeitiger Vollbelastung durch den Patienten, deren Compliance häufig herabgesetzt ist, dar.

\section{Minimalinvasive Osteosynthesen}

Bei ausgeprägter Osteoporose muss die Osteosynthese epi- und metaphysärer Extremitätenfrakturen häufiger als minimalinvasives Verfahren durchgeführt werden. Ein typisches Beispiel hierfür ist die Spickdrahtosteosynthese der distalen Radiusfraktur und die perkutane Osteo-
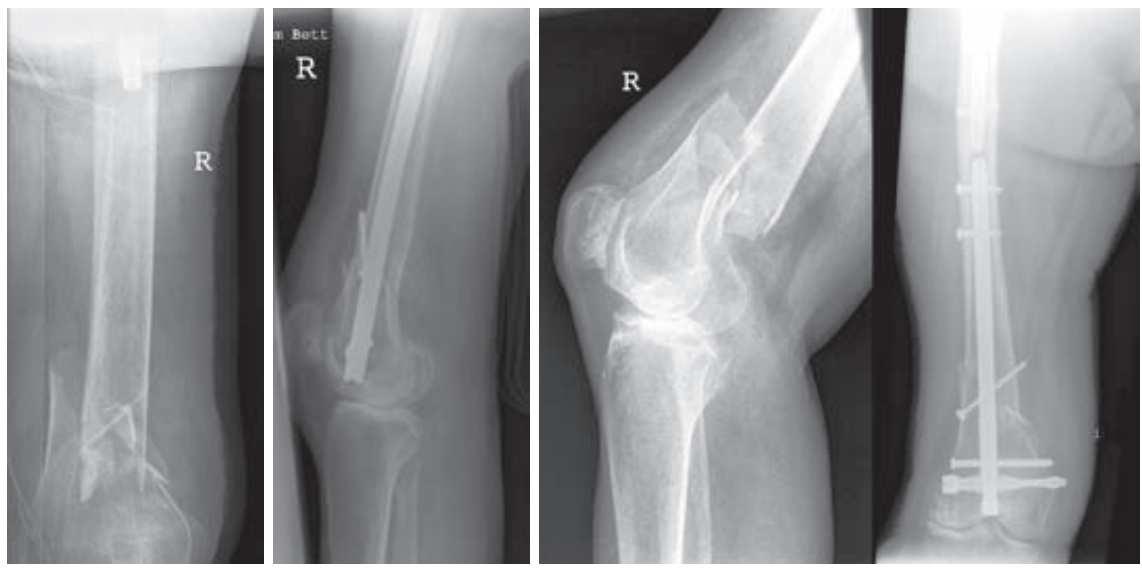

Abb.2a Distale Femurfraktur und Versorgung mittels distalem Femurnagel (DFN).

synthese mit Schrauben/Spickdrähten des Humeruskopfes (Abb.1). Bei komplexeren Frakturen mit ausgeprägter Trümmerzone sowie bei Frakturen mit Gelenkbeteiligung steht weiterhin der Fixateur externe als minimalinvasives Verfahren zur Verfügung. Ihnen gemeinsam ist die prinzipielle Möglichkeit der Anlage in Lokal- oder Regionalanästhesie zur Reduzierung der allgemeinnarkoseassoziierten Morbidität, die kurze Operationszeit und die geringe operationsbedingte Traumatisierung der umgebenden Weichteilstrukturen. Nachteilig ist ihre Beschränkung auf bestimmte Frakturtypen sowie die verminderte Primärstabilität der Osteosynthese. Dies macht häufig zusätzlich zur Operation eine an und für sich unerwünschte Immobilisation des betroffenen Skelettabschnitts für 4-6 Wochen notwendig.

\section{Intramedulläre Kraftträger}

Diaphysäre und diametaphysäre Frakturen der langen Röhrenknochen können mittels intramedullärer Stabilisierung zur Ausheilung gebracht werden. Typische Beispiele hierfür sind die Verriegelungsmarknagelosteosynthesen an Humerusschaft, proximalem und distalen Femur und Tibiadiaphyse (Abb.2a u. b). Dabei wird eine gute Primärstabilität durch die zentrale Krafteinleitung über den intramedullären Kraftträger erreicht. Die mangelnde Rotationsstabilität muss jedoch durch Verriegelungsschrauben optimiert werden, die in den von der Osteoporose besonders befallenen Knochenregionen zu liegen kommen. Dies äußert sich in einer vermehrten Komplikationsrate durch Schraubenlockerung, Schraubenwanderung und dem sogenannten Cut-OutPhänomen. Weiterhin werden bei Patienten mit Osteoporose und gesteigerter Fallneigung auch Anschlussfrakturen an

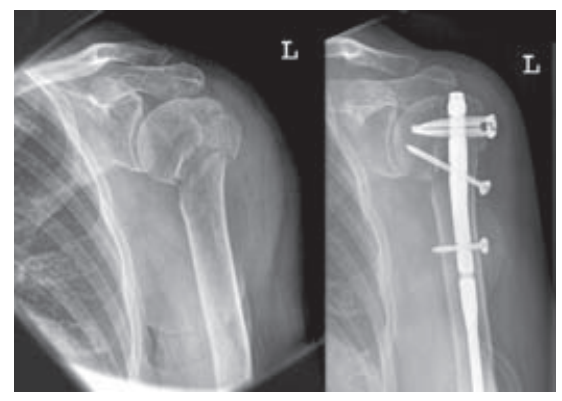

Abb.2b Humeruskopffraktur und Versorgung durch einen proximalen Humerusnagel (PHN).

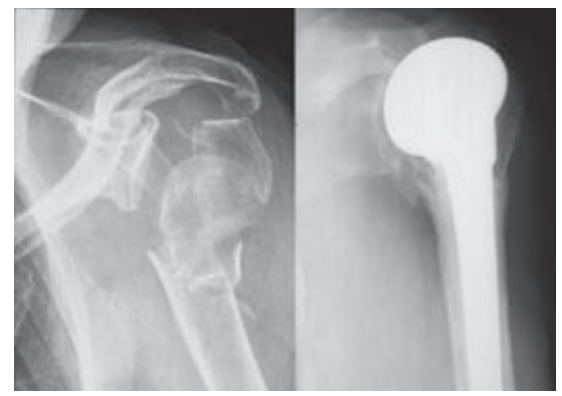

Abb. 3 Humeruskopf-Mehrfragmen-Fraktur mit Head-split und Versorgung mittels Schulterprothese.

den Enden der Implantate, mit der Folge aufwändiger Folgeeingriffe, beobachtet.

\section{Prothesen}

Für epi- und metaphysäre Frakturen höherer Schweregrade ist daher die Endoprothetik häufiger als bei Patienten in jüngerem Lebensalter die Methode der Wahl. Schenkelhalsfrakturen, pertrochantäre Frakturen und Drei- oder Vierfragmentfrakturen des Humersukopfes seien hierfür als Beispiele genannt (Abb.3). Mit (zementierten) Endoprothesen ist eine sofortige Übungs- und Belastungsstabilität gewährleistet. 


\section{Winkelstabile Implantate}

Grundproblem bei der operativen Therapie osteoporotischer Knochenbrüche stellt neben dem z.T. hohen Alter der Patienten die Minderung der Knochenfestigkeit, ausgelöst durch die Rarefizierung der spongiösen Knochensubstanz, dar. Dies resultiert in einer schwierigeren Verankerung der Osteosynthese und einer Reduzierung der Primärstabilität. Neben den bereits genannten Osteosyntheseverfahren wurden daher neue Implantate entwickelt, deren besserer Halt im osteoporotischen Knochen eine belastungsfähige Primärstabilität ermöglichen soll. Das Funktionsprinzip der winkelstabilen Implantate gleicht dabei dem des Fixateur externe. Durch eine stabile Verbindung zwischen dem extramedullären Kraftträger (Platte) und den Kraftaufnehmern (Schrauben) soll eine mögliche Angulation zwischen den Schrauben und der Platte verhindert werden. Die Winkelstabilität wird dabei durch ein den Schraubenkopf mit einbeziehendes Gewinde erreicht. Vor allem bei der Osteosynthese distaler Radius- und proximaler Humerus-(Kopf-)Frakturen fanden die winkelstabilen Platten den Eingang in die unfallchirurgische Routine (Abb.4). Ein signifikanter Unterschied zur herkömmlichen Plattenosteosynthese konnte bisher jedoch in keiner klinischen Studie gezeigt werden. Die beschriebene dreifach höhere Primärstabilität insbesondere im osteoprorotischen Knochen ist das Resultat biomechanischer Untersuchungen an Leichenpräparaten [5].

\section{Verbundosteosynthese}

Das Konzept der Verbundosteosynthese, also die Kombination eines Osteosyntheseverfahrens mit der zusätzlichen Applikation von Knochenzement findet in der Therapie pathologischer Frakturen eine breite Anwendung. Hierbei übernimmt der eingebrachte Knochenzement die Tragefunktion des metastatischen Knochengewebes. Dadurch wird eine ausrei-

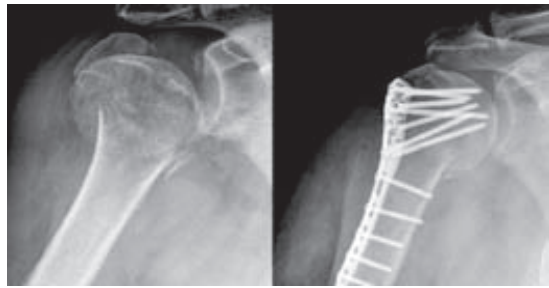

Abb. 4 Humeruskopffraktur und Versorgung mittels winkelstabiler Plattenosteosynthese (Philos).

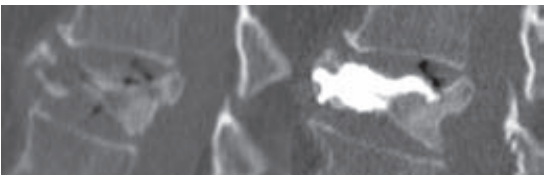

Abb.5 Wirbelkörperkompressionsfraktur und Versorgung mittels Vertebroplastie.

chende Belastbarkeit des befallenen Knochenabschnitts wiederhergestellt, eine Entlastung der Extremität ist nicht notwendig. Auch bei der osteoporoseassoziierten Fraktur kommt dieses Verfahren in Einzelfällen zur Anwendung, denn bei osteoporotischen Knochenbrüchen konnte in experimentellen Untersuchungen eine deutliche Verbesserung der Primärstabilität durch die Kombination herkömmlicher Osteosyntheseverfahren und der Zementapplikation vor allem am Schenkelhals und am distalen Radius gezeigt werden.

\section{Vertebroplastie und Kyphoplastie}

Die Wirbelkörperkompressionsfraktur stellt die häufigste osteoporotische Fraktur dar.

Das Auftreten einer Wirbelfraktur hat einschneidende Konsequenzen für den betroffenen Patienten. Es konnte gezeigt werden, dass die Morbidität von vier Wirbelkörperfrakturen der Morbidität einer hüftgelenknahen Femurfraktur entspricht $[10 ; 11]$. Neben der konservativen Therapie mit adaptierter medikamentöser Analgesie, möglichst kurzer Ruhigstellung, folgender Physiotherapie und der Anwendung von Orthesen [13], steht bei persistierender Schmerzsymptomatik über 4-6 Wochen die operative Therapie. Die bisherigen Standardverfahren wie ventrale Spondylodese, Wirbelkörperersatz oder dorsoventrale Stabilisierung, bedeuteten für die Patienten einen großen Eingriff mit entsprechenden Risiken. Dies gilt insbesondere in einer Population mit einer großen Anzahl ASA 3 und ASA 4 klassifizierter Patienten. Eine vergleichsweise neue und wenig belastende Therapieoption bei osteoporotischen Wirbelkörperfrakturen stellt die

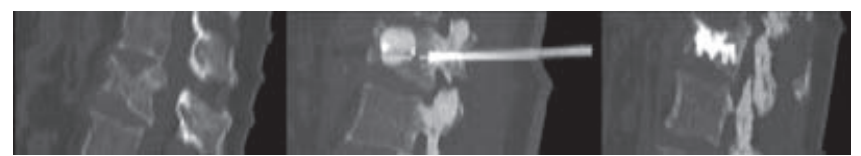

Abb.7 Wirbelkörperkompressionsfraktur und Versorgung mittels Ballon-Kyphoplastie.

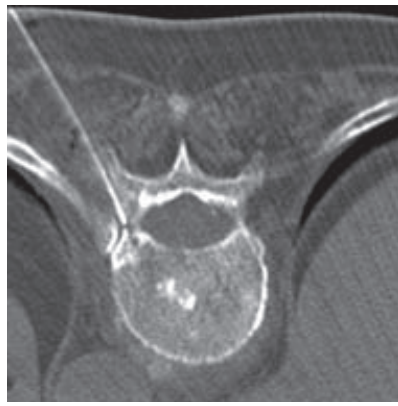

Abb. 6 Dorsale, transpedikuäre Punktion eines Wirbelkörpers zur Vertebroplastie oder Kyphoplastie.

Vertebro- bzw. Kyphoplastie dar. Bei der Vertebroplastie werden unter fluoroskopischer Kontrolle 2-4 ml eines PMMAKnochenzements in den frakturierten Wirbelkörper injiziert (Abb.5). Dies geschieht über einen transpedikulären $\mathrm{Zu}-$ gang, oder, vor allem in der Brustwirbelsäule, durch direkte, dorsale Punktion des betroffenen Wirbelkörpers (Abb.6).

Bei der Kyphoplastie wird der Wirbelkörper im Gegensatz zur Vertebroplastie zuvor mittels eines Ballonkatheters und der Installation eines Kontrastmittels unter hohen Drücken wieder aufgerichtet (Abb. 7).

Der Knochenzement wird sodann in die damit präformierte Höhle eingebracht. Dies führt zu einer Stabilisation des frakturierten Wirbelkörpers und $\mathrm{zu}$ einer Reduktion schmerzhafter Mikrobewegungen. Außerdem verhindert der Knochenzement das weitere Zusammensintern des betroffenen Wirbelkörpers.

Im Vergleich zur herkömmlichen Stabilisierung schmerzhafter Wirbelsäulensegmente mittels dorsaler und/oder ventraler Spondylodese zeichnen sich beide Methoden durch ihre rasche Durchführbarkeit, geringe Traumatisierung der Rückenmuskulatur und seltene Komplikationen aus. Weiterhin besteht die Möglichkeit der Durchführung in Lokalanästhesie oder unter alleiniger Analgosedierung. Eine Intubationsnarkose ist in den seltensten Fällen notwendig. Der stationäre 
Aufenthalt ist auf wenige (2-3) Tage beschränkt, die Patienten sind sofort nach dem Eingriff voll mobilisierbar und eine Rehabilitation ist nicht notwendig.

Beiden Interventionen gemeinsam ist eine rasche und anhaltende Schmerzreduktion, die häufig bereits innerhalb der ersten 48 Stunden zu beobachten ist $[4 ; 6]$. Die Komplikationsrate beider Verfahren wird in der Literatur mit 1-3\% angegeben, es werden Zementaustritte in die paravertebralen Venen und in den Spinalkanal sowie Anschlussfrakturen der benachbarten Wirbelkörper beschrieben [7-9].

\section{Therapie der Grundkrankheit}

Neben der Behandlung der Fraktur steht analog zu der Situation bei anderen pathologischen Frakturen die Therapie der Grundkrankheit. Neben einer Reduktion der Risikofaktoren (niedriges Körpergewicht, Rauchen, wenig Bewegung) und der physikalischen Therapie steht dabei am Anfang eine suffiziente Schmerztherapie, gefolgt von der sog. Basistherapie mit 1000 mg Kalzium und 1000 IE Vitamin $D_{3}$ pro Tag. Weiterhin steht uns heute eine Reihe von Medikamenten zur Verfügung, die im Wesentlichen in zwei Gruppen aufgeteilt werden können:

Antiresorptive Substanzen:

- Raloxifen, Bisphosphonate, Östrogene,

- Östrogen/Gestagen und Tibolon

Osteoanabole Substanzen:

- Parathormon, Strontium

Die Effektivität der genannten Substanzen wird heute nicht mehr über die messtechnische Zunahme der Knochendichte, sondern allein über die Reduktion des Frakturrisikos bestimmt. Für Bisphosphonate, Raloxifen wie auch für Parathor- mon und Strontium ist eine Reduktion des Folgefrakturrisikos vetrebraler und extravertebraler Frakturen von 50-70\% belegt [16].

\section{Ausblick}

Zukünftig liefern die experimentelle Unfallchirurgie und die Biomechanik neue Möglichkeiten zur Stabilisierung osteoporotischer Frakturen. Neben der Weiterentwicklung bewährter Osteosyntheseverfahren wird hierbei besonderer Wert auf die Entwicklung biodegradierbarer Knochenersatzstoffe als Ersatz für den PMMA-Knochenzement und die Optimierung des Implantatdesigns gelegt.

Den wichtigsten Meilenstein für eine zeitgemäße Versorgung der betroffenen Patienten stellt jedoch unseres Erachtens das Begreifen der osteoporotischen Fraktur als Symptom einer generalisierten Skeletterkrankung dar.

\section{Literatur}

${ }^{1}$ Adler CP. Knochenkrankheiten. Thieme, Stuttgart, New York 1983

2 Bartl R. Osteoporose - Prävention, Diagnostik, Therapie. Thieme, Stuttgart New York 2001

3 Bartl R, Bartl C, Mutschler W. Diagnostik und Therapie der Osteoporose. Unfallchirurg 2003; 106: 526-541.

${ }^{4}$ Chiras J, Depriester C, Weill A, Sola-Martinez MT, Deramnd H. Percutaneous vertebral surgery: technics and indications. J Neuroradiol 1997; 24: 45-59

${ }^{5}$ Claes L. Das Prinzip der Winkelstabilität in der Osteosynthese. OP-Journal 2004; 20: 471332

${ }^{6}$ Deramond H, Depriester C, Galibert P Le Gars D. Percutaneous vertebroplasty with polymethylmethacrylate: technique, indications, and results. Radiol Clin North Am 1998; 36 : 533-546

7 Franck H, Boszczyk B, Bierschneider $M$ Jaschke H. Interdisciplinary approach to balloon kyphoplasty in the treatment of osteoporotic vertebral compression fractures. Eur Spine J 2003; 12: 163-167
8 Gangi A, Kastler BA, Dietmann JL. Percutaneous vertebroplasty guided by a combination of CT and fluoroscopy. AJNR Am J Neuroradiol 1994; 15: 83-86

${ }^{9}$ Grados F, Depriester C, Cayrolle G. Long-term observations of vertebral osteoporotic fractures treated by percutaneous vertebroplasty. Rheumatology 2000; 39: 1410-1414

10 Matthis C, Weber U, O'Neill TW, Raspe H. Health impact associated with vertebral deformities: results from the European Vertebral Osteoporosis Study (EVOS). Osteoporos Int 1998; 8: 364-72

${ }^{11}$ Melton LJ. Excess mortality following vertebral fracture. J Am Geriattr Soc 2000; 48: 338-9

12 Mirels H. Metastatic disease in long bones. Clin Orthop 1989; 249: 256

13 Pfeifer M, Begerow B, Minne HW. Effects of a new spinal orthosis on posture, trunk strength, and quality of life in women with postmenopausal osteoporosis: a randomized trial. Am J Phys Med Rehabil. 2004; 83: 17786.

14 Report of a WHO Scientific Group, Prevention and Management of Osteoporosis, WHO Technical Report Series 2003; No 921

15 Vogel T, Dobler T, Bitterling H et al. Osteoporose in der Unfallchirurgie: Prävalenz und Management. Unfallchirurg 2005; 108: 356364

16 Vogel T, Kampmann P, Bartl R, Pfeifer KJ, Mutschler W. Osteoporose - Diagnostik und Therapie. OP Journal 2005; 16: 154-159

17 World Health Organization. Assessment of fracture risk and its application to screening for postmenopausal osteoporosis. Technical Report Series. WHO, Genf. 1994

\section{Tobias Vogel}

Assistenzarzt

Leiter der Arbeitsgruppe

Klinisches Osteoporose-Management Prof. Dr. med. Wolf Mutschler Direktor

Chirurgische Klinik und Poliklinik der Universität München-Innenstadt Nussbaumstrasse 20 80336 München 